\title{
Orientações para idosas que cuidam de idosos no domicílio
}

\author{
TEACHING PROGRAM FOR ELDERLY WOMEN WHO CARE FOR ELDERLY RELATIVES IN \\ THEIR HOME: REPORT OF EXPERIENCE
}

\author{
ORIENTACIONES PARA MUJERES ANCIANAS QUE CUIDAN DE FAMILIARES ANCIANOS \\ EN SU DOMICILIO: RELATO DE EXPERIENCIA
}

\section{Maria José D’Elboux Diogo1, Maria Filomena Ceolim², Fernanda Aparecida Cintra ${ }^{3}$}

\begin{abstract}
RESUMO
Trata-se de um relato de experiência de um programa de orientação para idosas cuidadoras de idosos no domicílio, realizado pelo Grupo de Atenção à Saúde do Idoso (GRASI), do Hospital de Clínicas da Universidade Estadual de Campinas. Esse programa foi elaborado e desenvolvido para atender uma solicitação de idosas cuidadoras, e participantes do GRASI. Ao final das atividades, as participantes relataram que o programa possibilitou o melhor enfrentamento das dificuldades, a compreensão das distintas alterações presentes na velhice, e o desenvolvimento de procedimentos que facilitam a relação com o idoso no dia-a-dia.
\end{abstract}

\section{DESCRITORES}

Cuidadores.

Idoso.

Educação em saúde.

Saúde do idoso.

\begin{abstract}
This is a report of experience about the development of a program designed especially for the orientation of elderly people who care for elderly relatives at home. It was carried out by the coordinators of the Group of Attention for Elderly People's Health (GRASI), of the Campinas State University's Clinics Hospital. This program was developed based on the needs reported by elderly caregivers who take part in the activities of the GRASI. The participants reported that the program made coping with the difficulties related to caregiving better; that there had been an improvement in the understanding of the changes brought by the aging process; and that it made possible the development of procedures that make easier the daily relationship with the elderly that is being cared for.
\end{abstract}

\section{KEY WORDS}

Caregivers.

Aged.

Health education.

Aging health.

\section{RESUMEN}

Este artículo es el relato de experiencia de un programa de orientación a mujeres ancianas que cuidan de familiares ancianos en su domicilio. Este programa fue desarrollado por el Grupo de Atención a la Salud del Anciano (GRASI), del Hospital de las Clínicas de la Universidad Estatal de Campinas. El objetivo del programa fue atender a la solicitud de mujeres ancianas cuidadoras y que participaban del GRASI. Al final de las actividades del mismo, las cuidadoras relataron que el programa posibilitó un mejor enfrentamiento de las dificultades vividas, una mejor comprensión de los cambios que suceden en la vejez y el desarrollo de procedimientos que favorecen la relación cotidiana con el anciano.

\footnotetext{
1 Enfermeira. Professor Associado do Departamento de Enfermagem. Faculdade de Ciências Médicas. Universidade Estadual de Campinas FCM/UNICAMP. mariadio@uol.com.br 2 Enfermeira. Professor Assistente Doutor do Departamento de Enfermagem. FCM/UNICAMP. fceolim@fcm.unicamp.br 3 Enfermeira. Professor Assistente Doutor do Departamento de Enfermagem. FCM/UNICAMP. fernanda@fcm.unicamp.br
}

\section{DESCRIPTORES}

Cuidadores.

Anciano.

Educación en salud.

Salud del anciano. 
Maria José D'Elboux Diogo Maria Filomena Ceolim Fernanda Aparecida Cintra

\section{INTRODUÇÃO}

A capacitação de recursos humanos especializados e o apoio ao desenvolvimento de cuidados informais compreendem algumas das diretrizes essenciais para o alcance do propósito da Política Nacional de Saúde do Idoso ${ }^{(1)}$.

No que diz respeito à capacitação de recursos humanos especializados, a Política prevê uma integração entre os serviços de saúde e as instituições de ensino profissional e superior, bem como a articulação do Ministério da Educação e as instituições de ensino superior, por intermédio de Centros Colaboradores de Geriatria e Gerontologia. Essa capacitação visa o preparo de recursos humanos para a operacionalização de atividades que incluem: a prevenção de perdas, a recuperação e a manutenção da capacidade funcional da população idosa, e o controle dos fatores que interferem na saúde dessa população.

No que confere ao apoio para o desenvolvimento de cuidados informais, a Política Nacional de Saúde do Idoso ${ }^{(1)}$ prevê uma parceria entre os profissionais da saúde e as pessoas próximas aos idosos, responsáveis pelos cuidados diretos. A parceria configura-se numa estratégia atual e menos onerosa que visa promover e manter a capacidade funcional dos idosos dependentes ${ }^{(2)}$. Essa parceria atende, ainda, a demanda de instrumentalização dos familiares para o cuidado do idoso e para a atenção à própria saúde, tendo em vista que a tarefa de cuidar/cuidado do idoso dependente é desgastante e constitui fator de risco à saúde do próprio cuidador.

\section{Considerando os Cuidadores de idosos}

No contexto familiar, a função de cuidador tende a ser assumida por uma única pessoa, denominada "cuidador principal"(3-4). Essa pessoa assume e se responsabiliza pelas tarefas de cuidado, sem contar, na maioria das vezes, com a ajuda de outro membro da família ou de profissionais. Representa, ainda, o elo entre idoso/família e a equipe de saúde ${ }^{(5)}$.

A designação do cuidador é informal e resulta de uma dinâmica, embora possa parecer que esse processo atende certas regras fundamentadas em quatro fatores ${ }^{(6)}: 1$. parentesco (em sua maioria, os cônjuges); 2. gênero (predominantemente a mulher); 3. proximidade física (quem convive com o idoso); e, 4. proximidade afetiva (estabelecida pela relação conjugal e pela relação entre pais e filhos).
Pesquisas nacionais e internacionais apontam que os cuidadores são, em uma hierarquia, as esposas, a filha mais velha ou a nora mais velha, e a filha solteira ou viúva. Assim, a mulher evidencia-se como a "grande cuidadora", a quem foi atribuído esse papel cultural e socialmente, ao cuidar dos filhos, do marido, dos doentes e dos velhos ${ }^{(3)}$. A esse respeito, afirma-se que a experiência de cuidar de familiares sugere para a mulher uma "carreira de cuidado"(7).

Apesar do papel de "grande cuidadora" atribuído à mulher, decorrente das mudanças estruturais da família atual, da inserção da mulher no mercado de trabalho, e do grau de incapacidade do idoso, um outro membro da família colabora no cuidado. Esse elemento é denominado "cuidador secundário" e cuida ocasionalmente, sem o compromisso e a responsabilidade formal pelo cuidado. Ele divide a responsabilidade com o cuidador principal, auxiliando-o e substituindo-o quando necessário, fornece retaguarda financeira ou ajuda em situações específicas como, por exemplo, no transporte, nas atividades sociais, e nas atividades financeiras ${ }^{(3,7-8)}$.

A dinâmica da atividade de cuidar no domicílio pode, contudo, gerar uma ambigüidade identificada pelo bem-estar e pela tensão entre os familiares. O bem-estar é gerado nas situações em que os recursos familiares atendem a demanda de cuidados ${ }^{(9)}$. Por outro lado, quando esses recursos são insuficientes, a demanda da própria família pode desencadear tensão no contexto familiar, pelo aumento dos conflitos entre os seus membros, pela falta de resolução dos problemas, e pelas tarefas não concluídas.

$\mathrm{Na}$ literatura, a maioria dos estudos vincula ao papel do cuidador ônus e estresse, segundo a avaliação dos familiares que desempenham esse papel. Pesquisadores ${ }^{(10)}$ destacam os seguintes fatores estressantes, relatados por cuidadoras de idosos: 1. os cuidados diretos, contínuos, intensos, e a necessidade de vigilância constante; 2 . o desconhecimento ou a falta de informações para o desempenho do cuidado; 3 . a sobrecarga de trabalho para um único cuidador, especialmente os problemas de saúde desencadeados pela idade avançada da cuidadora; 4. a exacerbação ou o afloramento de conflitos familiares, vinculados ao trabalho solitário do cuidador (sem ajuda) e ao não reconhecimento por parte dos demais familiares; 5 . a dificuldade para adaptar as demandas da situação de cuidado aos recursos disponíveis, incluindo os recursos financeiros, a redução das atividades sociais e profissionais, o abandono das atividades de lazer, entre outros. 
Entretanto, uma nova tendência de pesquisa pode ser observada a partir do final da década de 1980, a qual identifica aspectos positivos ou benéficos vivenciados pelos cuidadores. Estes aspectos também relatados por cuidadores, relacionamse a: 1 . crescimento pessoal; 2 . aumento do sentimento de realização, do orgulho e da habilidade para enfrentar desafios; 3. melhora no relacionamento interpessoal, tanto com o idoso quanto com as outras pessoas; 4. aumento do significado da vida; 5. prazer; 6. satisfação; 7. retribuição; 8. satisfação consigo próprio; e, 9. bem-estar com a qualidade do cuidado oferecido ${ }^{(7)}$.

Apesar dos estudos mostrarem relações afetivas positivas no cuidar/cuidado do idoso, a problemática vivenciada pelos cuidadores revela a necessidade de incremento das modalidades de apoio aos cuidadores familiares, por meio dos programas de atendimento domiciliário, dos serviços de cuidador substituto, bem como dos serviços de informação, orientação, encaminhamento e apoio dos profissionais da área de saúde.

\section{O cuidador idoso no cenário domiciliar}

Estudos mostram que a esposa é, com freqüência, a cuidadora primária de idosos, seguida da filha que também possui idade avançada. Desta forma, pessoas que vivenciam o envelhecimento, ou que já se encontram em plena velhice, assumem a tarefa de cuidar, embora apresentem alguma alteração na capacidade funcional e até mesmo na sua saúde.

Nos Estados Unidos da América 60\% das cuidadoras primárias de idosos são esposas, e 73\% delas possuem idade igual ou superior a $65 \operatorname{anos}^{(11)}$. Estas pessoas são chamadas "vítimas ocultas" pois convivem com o isolamento, a solidão, e a sobrecarga de funções. Além da responsabilidade pelo cuidado nas 24 horas diárias, elas enfrentam o seu próprio envelhecimento e o comprometimento físico, bem como as responsabilidades financeiras e legais. Estas questões interferem no relacionamento conjugal, desencadeiam situações estressantes e problemas de saúde nas cuidadoras.

Dados da literatura mostram que os grupos de suporte e os programas educativos oferecem benefícios para os cuidadores, como a redução do estresse, da depressão e da ansiedade. Outros trabalhos, contudo, reconhecem que essas atividades não produzem efeitos significativos sobre as reações negativas, mas causam impacto sobre os elementos mediadores do estresse e do bem-estar do cuidador. Para exemplificar, as orientações sobre as condições de saúde do idoso dependente, a instrumentalização do cuidador para o próprio cuidado e para o enfrentamento da situação, o suporte social e as orientações sobre os recursos da comunidade disponíveis, são alguns dos resultados comumente obtidos. Tais orientações não agem diretamente sobre o estresse do cuidador, mas, podem reduzir a ocorrência de fatores desencadeantes do estresse, como o desconhecimento sobre o cuidado a ser realizado ${ }^{(12)}$.

Um dos conceitos-chave na ação da enfermeira gerontóloga: os cuidadores necessitam de treinamento (orientações, ações educativas), pausa na função de cuidador, e de cuidado a si próprios $^{(13)}$.

Identificam-se três tipos de programas de suporte direcionados aos cuidadores: os Grupos de apoio conduzidos; os Grupos de treinamento conduzidos profissionalmente; e a Psicoterapia/ aconselhamento (acolhimento) ${ }^{(12)}$. Estes programas têm os seguintes objetivos: 1. reduzir o isolamento dos cuidadores; 2. mobilizar os recursos e suportes para ajudar o cuidador; 3 . aumentar o conhecimento sobre o processo de envelhecimento e os problemas de saúde específicos desta faixa etária; 4. melhorar o desempenho do cuidador; 5. aumentar a capacidade de resolução de problemas e o desenvolvimento de habilidades; e, 6. direcionar os problemas emocionais e de relacionamento que podem emergir no cuidado. Em síntese, esses programas visam o bem-estar físico e emocional do cuidador, e a redução da sua sobrecarga, a qual representa um dos elementos mediadores do estresse, conforme citado anteriormente. Em algumas situações, eles contribuem, ainda, para retardar a institucionalização do idoso fragilizado ou dependente.

A esse respeito, as reuniões em grupos, e particularmente os programas educativos, representam recursos valiosos, uma vez que oferecem oportunidades para os cuidadores expressarem os seus sentimentos, as dificuldades vivenciadas, as expectativas em relação à sua pessoa e ao idoso dependente no contexto domiciliar, entre outras carências.

\section{OBJETIVO}

O presente trabalho tem o objetivo de apresentar e discutir um programa de educação em saúde para idosas que cuidam de idosos no domicílio.

\section{TRAJETÓRIA METODOLÓGICA}

O programa de educação em saúde para as cuidadoras de idosos foi desenvolvido no Grupo
Orientações para idosas que cuidam de idosos no domicílio 
Maria José D'Elboux Diogo Maria Filomena Ceolim Fernanda Aparecida Cintra de Atenção à Saúde do Idoso (GRASI). Esse Grupo desenvolve atividades de ensino, assistência e pesquisa desde março de 1997, e está vinculado ao Ambulatório de Medicina Interna do Hospital de Clínicas da Universidade Estadual de Campinas - UNICAMP ${ }^{(14)}$.

No final de 1999, oito idosas participantes do GRASI, há aproximadamente dois anos, solicitaram às coordenadoras desse Grupo, e autoras do presente estudo, orientações sobre como cuidar de pessoas acamadas, particularmente dos idosos. As justificativas apontadas relacionavamse às dificuldades vivenciadas no cuidado de seus familiares (pai, mãe, esposo, irmão, irmã) acamados ou dependentes.

Inicialmente, elaboramos um formulário, com questões abertas e fechadas, para a caracterização dessas idosas e a identificação das suas experiências e expectativas, visando a elaboração de um programa que melhor atendesse as suas necessidades. Foi-lhes solicitado a responder as perguntas, sendo-lhes esclarecido que as informações obtidas seriam necessárias para o melhor desenvolvimento da proposta por elas apresentada. Embora não se tratasse de um trabalho de investigação, foi-lhes assegurado o anonimato. O formulário foi aplicado individualmente pelas autoras do presente estudo, em uma das reuniões do GRASI, às oito idosas que constituíram o grupo de trabalho.

O número de participantes $(\mathrm{n}=8)$ mostrouse adequado para o desenvolvimento do programa, uma vez que possibilitou a emergência das emoções, a troca de experiências, o confronto, a negociação e a partilha entre os pares ${ }^{(15)}$. Um número superior a esse poderia inviabilizar a nossa proposta de trabalho junto às idosas, pela inibição de algumas em detrimento de outras que apresentassem maior capacidade de expressão e de controle sobre o grupo.

As idosas pertenciam à faixa etária entre $56 \mathrm{a}$ 75 anos. Todas tinham pelo menos o primeiro grau incompleto; uma havia completado o segundo grau. A maioria $(\mathrm{n}=7)$ era casada ou amasiada; uma era viúva. Quatro idosas residiam junto ao idoso que recebia os cuidados. Com respeito ao grau de parentesco com esses idosos, quatro eram esposas (dentre elas, uma também cuidava do cunhado); uma era cunhada; uma era filha que cuidava da mãe e do pai; e duas eram irmãs.

Dentre os motivos apontados pelas idosas para os cuidados dos familiares destacaram-se: afecções como diabetes, depressão, doença de
Alzheimer, epilepsia, e déficits como a presbiacusia e a dependência para o autocuidado. Uma idosa afirmou que, com freqüência, era procurada por vizinhos e amigos para auxiliar no cuidado de outras pessoas.

Ao serem solicitadas a manifestar sobre a mudança na vida, enquanto cuidadoras, cinco relataram que a vida mudou desde que começaram a cuidar do idoso no domicílio. Os relatos mais significativos foram:

Tem que ter mais cuidado com a alimentação...

(57anos, cuida da mãe e do pai);

... a maneira de ver a necessidade do

paciente (73 anos, cuida do irmão);

A (minha) vida mudou porque vivo em função dele (marido) (53 anos, cuida do esposo);

Esses cuidados são a razão da minha vida! (68 anos, cuida do esposo e de outras pessoas, quando solicitada).

As principais dificuldades vivenciadas pelas idosas no cuidado ao idoso no domicílio foram: disponibilidade de transporte para os Serviços de Saúde, resistência do idoso ao cuidado, ambiente inadequado para melhor atendimento, "falta de conhecimento" para o cuidado e para a distinção entre uma necessidade orgânica e uma necessidade emocional por carência afetiva, e "falta de paciência". Essas dificuldades aproximam-se dos fatores estressantes destacados, citados anteriormente ${ }^{(10)}$, e correspondem aos achados de outras investigações nacionais e internacionais.

Sobre as expectativas das idosas em relação ao programa foram apontadas: "aprender como lidar com a pessoa doente" (por exemplo, idosos que recusam a medicação), "saber as características de algumas doenças", "aprender a realizar cuidados" (como higiene corporal, alimentação, comunicação), "ampliar os conhecimentos gerais" e, em particular, sobre o idoso.

A partir dessas informações elaboramos o "Programa de orientação para familiares de idosos fragilizados", com o objetivo de fornecer subsídios a familiares de idosos para a realização do cuidado destes no domicílio, visando a atenção à saúde e à melhoria da qualidade de vida tanto dos idosos quanto dos cuidadores. A carga horária total foi de 36 horas, desenvolvida em reuniões mensais, no período de março de 2000 a outubro de 2001. 
Para o desenvolvimento do programa utilizamos a abordagem pedagógica de Paulo Freire, na qual a ação educativa fundamenta-se nas experiências vividas pelos sujeitos ${ }^{(16)}$. Nesse sentido, a nossa preocupação foi elaborar um programa que contemplasse as dificuldades apresentadas pelas idosas, e identificadas por meio do formulário, sem menosprezar as preocupações e os sentimentos que emergiam a cada encontro.

No programa foram abordados os seguintes temas: 1 . O processo de envelhecimento e a velhice (4h); 2. O papel do cuidador familiar (2h); 3. Doenças mais comuns na velhice (osteoporose, acidente vascular cerebral, hipertensão arterial e diabetes mellitus) (8h); 4. Transtornos do sono (2h); 5. Uso de medicamentos (2h); 6. Alimentação do idoso (2h); 7. Noções básicas de higiene (4h); 8 . Vestuário e cuidados com a pele (2h); 9 . Instabilidade e quedas $(2 \mathrm{~h}) ; 10$. Transferências e adaptações ambientais (4h); 11. Abusos e maus tratos (2h); e, 12. Relacionamento interpessoal (2h).

Os recursos audiovisuais utilizados na dinâmica com o grupo de idosas foram: retroprojetor, laboratório de enfermagem, lousa e giz, papel sulfite e lápis de cor, e recortes de revistas. Como estratégias, empregamos: a problematização e a vivência das idosas, o desenho livre, a exposição oral e atividades práticas.

Os trabalhos no grupo foram dinâmicos, produtivos e motivadores para os encontros subseqüentes. A cada encontro resgatavam-se os aspectos mais relevantes abordados no encontro anterior e a sua aplicação no cuidado do idoso. Essa dinâmica mobilizou as idosas para uma ação mais ampla e segura junto aos familiares, e a problematização de novas necessidades. Destaque foi dado à manutenção da autonomia e da independência do idoso fragilizado, e aos aspectos ergonômicos e posturais da pessoa que realiza os cuidados.

\section{DISCUSSÃO}

Ao final do programa realizamos uma avaliação com as participantes, as quais revelaram que ele atendeu às expectativas do grupo. Chamou-nos a atenção o seguinte depoimento da idosa de idade mais avançada do grupo (72 anos), ao ser solicitada para comentar sobre "como ajudar uma pessoa que necessita de cuidados": "Primeiro ... avaliar o grau de dependência, conversar, estabelecer contato com o toque das mãos, ter firmeza e cuidado". A atenção para a privacidade, para uma comunicação efetiva, para evitar a imposição do cuidado, para a valorização das capacidades, bem como com o respeito às limitações do idoso fragilizado e à preservação da sua autonomia e independência, emergiram em todos os encontros.

Dentre as sugestões feitas pelo grupo, destacaram-se: o oferecimento do programa semestralmente, o aumento da carga horária das atividades teóricas e práticas, e a inclusão de outros temas como "primeiros cuidados nas urgências" (engasgo, desmaio, entre outras, comumente, observadas em idosos).

Segundo as idosas, o programa ampliou o conhecimento de procedimentos que facilitam o lidar com o idoso no dia-a-dia e potencializou a capacidade de enfrentamento das dificuldades no cuidado no domicílio. Possibilitou, assim, uma melhor compreensão sobre como enfrentar as dificuldades e entender as distintas situações manifestas na velhice. Para elas, as atividades realizadas durante o programa contribuíram para a assistência a outros familiares, amigos e vizinhos.

A avaliação das idosas aproxima-se das investigações sobre os benefícios advindos ao cuidador na sua participação em grupos de suporte ${ }^{(17-20)}$.

O estudo da satisfação dos cuidadores após a sua participação em programa de treinamento e aconselhamento/acompanhamento, identificou maior número de aspectos positivos ${ }^{(12)}$. Apesar disso, os cuidadores apontaram limitações para a participação nas atividades, as quais estavam relacionadas à dificuldade de acesso à instituição do programa; às condições "para dar conta do trabalho"; às responsabilidades do cuidado do idoso e da família, concomitantemente; aos problemas de saúde do cuidador; e, à obtenção de um substituto para a sua ausência. Acreditamos que estas e outras limitações, embora não tenham sido identificadas pelo nosso grupo de idosas, poderiam ser minimizadas no envolvimento de toda a família, da equipe de saúde, dos membros da comunidade e do Estado.

Diante do exposto, a experiência vivenciada pelo programa remete a urgência da efetivação da Política Nacional de Saúde do Idoso ${ }^{(1)}$, para que programas semelhantes ao que apresentamos neste trabalho sejam desenvolvidos com regularidade e maior abrangência. Estes recursos poderão, ao menos, minimizar o quadro dramático dos idosos dependentes e fragilizados no domicílio.

\section{CONSIDERAÇÕES FINAIS}

Embora trate-se, ainda, de uma questão que gera questionamentos e inquietações, o cuidador
Orientações para idosas que cuidam de idosos no domicílio 
Maria José D'Elboux Diogo Maria Filomena Ceolim Fernanda Aparecida Cintra (idoso) é um elemento presente no cenário assistencial nacional. Esta realidade não pode ser omitida pela enfermeira gerontóloga, pelos órgãos governamentais e pela sociedade em geral. A atenção e o suporte a essas pessoas são fundamentais para a melhoria da qualidade de vida do idoso fragilizado e do próprio idoso cuidador.

\section{REFERÊNCIAS}

(1) Ministério da Justiça. Secretaria Nacional dos Direitos Humanos. Política Nacional do Idoso. Programa Nacional de Direitos Humanos. Brasília; 1998.

(2) Gordilho A, Sérgio J, Silvestre J, Ramos LR, Espíndola N. et al. Desafios a serem enfrentados no terceiro milênio pelo setor saúde na atenção integral ao idoso. Rio de Janeiro: UnATI; 2000.

(3) Wanderley MB. Publicização do papel do cuidador domiciliar. São Paulo: IEE/PUC; 1998. (Série Programas e Serviços de Assistência Social)

(4) Kawasaki K, Diogo MJD'E. Assistência domiciliária ao idoso: perfil do cuidador formal - Parte I. Rev Esc Enf USP 2001; 35:257-64.

(5) Duarte YAO, Diogo, MJD'E, organizadores. Atendimento domiciliar: um enfoque gerontológico. São Paulo: Atheneu; 2000. p.3-17.

(6) Mendes PBMT. Cuidadores: heróis anônimos do cotidiano. [dissertação] São Paulo (SP): Pontifícia Universidade Católica de São Paulo; 1995.

(7) Sommerhalder C. Significados associados à tarefa de cuidar de idosos de alta dependência no contexto familiar. [dissertação] Campinas (SP): Faculdade de Educação da UNICAMP; 2001.

(8) Pavarini SCI, Neri AL. Compreendendo dependência, independência e autonomia no contexto domiciliar: conceitos, atitudes e comportamentos. In: Duarte YAO, Diogo MJD'E, organizadores. Atendimento domiciliar: um enfoque gerontológico. São Paulo: Atheneu; 2000. p. 49-69.

(9) Fink SV. The influence of family resources and family demands on the strains and well-being of caregiving families. Nurs Res 1995; 44(3):139-45.

(10) Gonçalves LHT, Alvarez AM, Santos SMA. Os cuidadores leigos de pessoas idosas. In: Duarte YAO, Diogo MJD'E, organizadores. Atendimento domiciliar: um enfoque gerontológico. São Paulo: Atheneu; 2000. p.102-10.

(11) Hooyman N, Kiyak HA. Social gerontology - a multidisciplinar perspective. 4th ed. Needham Heights: Allyn and Bacon; 1996. The importance of social supports: family, friends, and neighbors; p. 282-95.
(12) Schofield H, Bloch S, Herman H, Murphy B, Nankervis J et al, editors. Family caregivers: disability, illness and ageing. Saint Leonards: Allen \& Unwin/Vichealth; 1998.

(13) Eliopoulos C. Gerontological nursing. 4th ed. Philadelphia: Lippincott; 1997. Family caregiving. p. $486-95$.

(14) Diogo MJD'E, Ceolim MF, Cintra FA. Implantação do Grupo de Atenção à Saúde do Idoso (GRASI) no Hospital de Clínicas da Universidade Estadual de Campinas (SP): relato de experiência. Rev Lat-Am Enferm 2000; 8(5):85-90.

(15) Cintra FA. A significação do glaucoma e a mediação dos significados de velhice na perspectiva vygotskiana: subsídios para a educação à saúde. Rev Esc Enferm USP 2000; 34:339-46.

(16) Freire P. Pedagogia do oprimido. $25^{\mathrm{a}}$ ed. Rio de Janeiro: Paz e Terra; 1998.

(17) Griffin MM. Caring for caregivers: a nursing role in a corporate setting. Geriatr Nurs 1993; 14 (4):200-4.

(18) Mittelman MS, Ferris SH, Shulman E, Steinberg G, Ambinder A, Mackell JA et al. A comprehensive support program: effect on depression in spouse-caregivers of AD patients. Gerontologist 1995; 35(6):792-802

(19) Brogna L. Caring for caregivers: home care nursing's challenge. In: Allender JA, Rector CL. Readings in gerontological nursing. Philadelphia: Lippincott, 1998. p. 515-22.

(20) Kaasalainen S, Craig D, Wells D. Impact of the caring for aged relatives group program: an evaluation. Public Health Nurs 2000; 17(3):169-77.

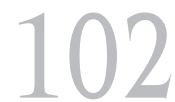

Rev Esc Enferm USP 2005; 39(1):97-102 